\title{
Peri urban agriculture as a new strategy of urban development: A case study in Cenaia, Pisa
}

\author{
B. Özel, S. Mecca, F.M. Lorusso \& L. Dipasquale \\ University of Florence, DIDA Department of Architecture, INN-LINKS Research Unit, Florence, Italy
}

\begin{abstract}
Rural-urban interface never vanishes but only slides outwards from the city core as the city grows. This interface, so-called "peri-urban area", is characterised by "dynamism" and "diversity" as it acts like the place where movements of people, commercial goods and capital take place. As a consequence, peri-urban area becomes the zone of coexistence of rural and urban areas, and constitutes a strong linkage between the two words. The Ecocity project in Cenaia aims to reorganize the expanding urban structure by giving a new identity to the margins of the city. The goal of the project is to valorize the urban fringe through an experimental co-farming/housing habitat and utilize peri-urban agriculture as its principle function. Furthermore the peri-urban area is revitalised by touristic, recreational and commercial activities. The aim of this paper is to point out the role of peri-urban agriculture that offers a symbiosis between urban and rural worlds.
\end{abstract}

\section{FOREWORD}

More than half of the world's population lives and works in the cities and this percentage keeps increasing each year. Since the 1950s, after the industrial revolution in Europa, urban growth became a global phenomenon. The cities have grown by three main reasons: "economic growth", "natural demographic increase" and "rural-urban migrations". Other effective factors that contributed to the rapid urban growth have been: natural disasters, lack of educational and medical facilities and poor infrastructure in rural areas. Obviously the reasons why the cities undergo these growths vary on the basis of their different geographical and historical features so it is difficult to define universal reasons for the urban growth.

People migration has always existed for many reasons, but the most significant one has always been economic. In the urban areas growing economy and new investment affairs attract people as they offer many employment opportunities and living comfort.

"Natural demographic increase" means the number of people being born each year compared to the number of deaths. This constitutes the main contributor reason to the growth of the cities.

"Migration" is another affecting factor of urban growth instead. After the industrial revolution up to the 1970s, "migration" has appeared as the movement of population from rural to urban areas. More industrial activities in the cities encouraged people to move from countryside to the cities to search for better living conditions and high-employment chances. Today the migration flow is not only seen from rural to urban areas but also happens at international levels.

The rapid urbanization brought unprecedented negative consequences such as urban poor, which is mainly formed in suburbs. The physical expansion of the cities occurs in the suburbs more than the central urban area. As a result of this, the cities and its suburbs spread over to the rural areas beyond their boundaries and this spread which is called "urban sprawl" causes loss of agricultural lands and eventually creates stress on the surrounding natural environment.

The increasing expansion of the urban footprint requires also effective ways to deal with many vital issues like food insecurity, protection of public and environmental health, resource management and land use planning. The "Peri-urban agriculture", as a strategy, meets with these issues in an innovative way by understanding better the dynamism of the peri-urban area.

\section{BACKGROUND AND THE NOTION OF PERI-URBAN AREA}

Peri-urban area is the term, which is used to describe the interface resulting from the process of peri-urbanisation. There are also other common terms that define this area such as rurban space, outskirts or hinterland. In addition to these terms, peri-urban area was thus seen as a landscape type 
formed by the interaction between urban and rural activities (Zasada, Fertner, Piorr \& Nielsen, 2011). The notion of peri-urban area has been interpreted in various conceptual manners. It is described as a heterogeneous settlement pattern at the urbanrural interface as a former model of an urbanrural dichotomy (Errington, 1994). A European perspective often sees peri-urban areas as mixed characterised zones created by urban influence but with a rural morphology (Caruso, 2001). In 2007 the Council of Europe defined the peri-urban interface as a transition zone moving from rural to urban. Different methods have been developed in order to define peri-urban areas; by MDP-ESA (Municipal Development Programme for Eastern and Southern Africa 2001) four main methods have been identified which base on:

- Physical criteria including street patterns and housing density.

- Functional criteria including employment levels and transportation networks.

- Social and socio-psychological criteria involving the determination of the urban life quality and the general social life.

- Administrative criteria covering the local authority boundaries.

As can be seen from the references there are limited concepts that define the peri-urban interface. The most common but erroneous understanding is to see peri-urban areas like simple peripheries, "border territories" of the city that manifests rural-urban features and accommodates only the urban poor. Moreover in economic terms peri-urban areas are seen as resources of low-price lands, which easily permits building industrial zones that requires larger areas. However, the significance of this dynamic linkage area, peri-urban interface, can not be reduced to the above listed limited perspectives. Peri-urban areas need to be understood as particular ecological and socio-economic ecosystems by observing the interactions and the dynamics of rural-urban linkage and flows. The fact that the peri-urban areas manifest mixity and coexistence of urban and rural characteristics, they require multidimensional approaches to be analysed and for a better understanding.

\subsection{Characteristics of Peri-urban area}

The belief that considers the border between urban and rural areas as a "cut line" in the landscape is increasingly getting abandoned because a clearly defined border can not exist in physical and functional terms. The interface between urban and rural areas are prone to a continual change due to the bidirectional movements (from urban to rural and vice versa) of people and the capital caused by urban growth, so it can be recognised that the urban and rural features coexist beyond their limits and the peri-urban area constitutes a strong linkage between these different worlds.

As a result of a constant transformation, the peri-urban areas gain a heterogeneous social and physical composition. The dwellers of peri-urban area can be characterised by both the lower income communities and the wealthier secondary house owners so the interface of rural and urban act like the meeting place of two different situations. This heterogeneity attributes to the peri-urban areas new features like "complexity", "dynamism" and "diversity"; furthermore, gives them the potential of being easily changed and transformed. Although the high potential of transformation of peri-urban areas seems like a positive feature, it may have negative impacts and vulnerabilities in the absence of clear regulations and efficient governance system in terms of environmental and social sustainability.

One of the issues that has a great role in determining the main characteristics of the peri-urban areas is having dichotomies in its nature as it always tends to gather conflictual situations like urban-rural, traditional-modern, cityside-countryside, richness-poverty. However, this conflictuality gives rise to the opportunities of co-existing with beneficial linkages and symbiosis (Zasada, Fertner, Piorr \& Nielsen, 2011).

\subsection{Interaction of rural-urban flows}

Due to a series of flows and migrations the ruralurban interface never vanishes but only slides outwards from the city core as the city grows. As mentioned before this interface is characterised by the movements of people, goods, capitals and natural resources. Such transformations due to these flows can be defined by the term of "periurbanisation" which is frequently seen as a result of post-modernity. Besides commercial and infrastructure developments, the internal migration flows represent the main reason to the peri-urbanisation. Internal migration is not a unidirectional movement but it happens in two ways: migration towards to city core and contrariwise. The last process is described by the term "counter-urbanisation" which indicates the migration flow from city core to the countryside.

Mitchell (2004) elaborated three different concepts and motivations that urge the counterurbanisation. The migrants move towards countryside for quite different reasons. In this context several researchers have defined "ex-urbanisation" as the migration of affluent people to the rural areas staying within commuting distances from the city. In the terms of ex-urbanisation the scope of 
migration is to have the amenities of living out of the city such as having a larger house, and garden for self food production. In this case the migrants keep their jobs and daily routines around their living places.

As the second concept of migration, "displace urbanisation"which is characterised by the migration of low-income groups due to the economic necessities such as affordable housing. This type of migration mainly refers to the young families who can not afford a house in the inner city. Displace urbanisation was seen especially in 1950s as the peri urban area was increasingly getting more attractive in the context of industrial revolution. The new employment opportunities and low-cost affordable housing features caused an intense migration from center to peri-urban areas and, as a result, abandonment of the historical city cores which with their limited and insufficient infrastructures were incapable to respond to the new demands of post-industrial communities. This population flux eventually has formed "dormitory suburbs". "Anti-urbanisation" represents the last version of counter-urbanisation concepts and it consists of the migration of the people who wants to escape from urban lifestyle and moves to the rural areas. This process is also called "retirement migration". As a result of all above mentioned migration motivations it can be seen that there is not only one way of flux of population so the urban area not only grows and intensifies but also can be de-concentrated by inner migrations.

\section{PERI URBAN AGRICULTURE AS A STRATEGY OF SUSTAINABLE URBAN DEVELOPMENT}

Agricultural systems can respond to both ecological and socio-economic needs of urban communities. The agricultural activities that are usually seen on the urban fringes are mainly the results of the absorption of the surrounding rural areas by the expanse urban footprint, so they don't constitute an intentional structured peri-urban agriculture system but are only the natural farming activities of rural settlements of pre-expansion zone.

"Urban agriculture", as it can be recognised by its name, refers to the agricultural activities that can be done in urban vegetable gardens, vacant plots, verges, balconies and terraces within the cities. Urban agriculture is mainly done for growing crops and raising small livestock for own consumption in the neighbourhoods. "Peri-urban agriculture" refers rather to the intensive semi or fully commercial agricultural production activities that take place in the farm units and fields close to the city in the rural-urban interface. Peri-urban agriculture includes also raising livestocks, fisheries and forestry productions.

\subsection{Main features of Peri urban agriculture}

What makes the peri urban agriculture more advantageous compared to rural agriculture is the "proximity" to the urban settlements that means people and potential of man work. The proximity can be seen as a beneficial situation but it can have also negative outcomes and risks. The opportunities consist of:

- availability of fresh, perishable food;

- easy access to food for urban poor;

- employment and income possibilities;

- waste management and recycling of urban waste water;

- less need of packaging and transportation.

Whereas the risks include:

- increased competition for land, water and labour; - reduced environmental capacity for absorption of the pollution.

If the production processes can be monitored and well controlled, peri-urban agriculture can provide numerous advantages in social and economic terms. However, peri-urban agriculture should not be in competition with rural agriculture as the production in peri-urban areas has different priorities like providing food security and access to the fresh food to the citizens before reaching to the commercial levels of production.

\subsection{Issues of Peri urban agriculture}

Peri-urban agriculture can contribute to improve various urban complications of economic, social and cultural dimensions when carried out properly under safe conditions. It has a considerable contribution to the food security of the urban communities. Firstly peri-urban production activities increase the quantity of available food. Urban poor often has a lack of purchasing capacity to acquire sufficient amount of food. Peri-agriculture appears to reduce food insecurity by providing direct access from home to self-produced food.

The peri-urban agriculture has been prevalently used as a local food production strategy in Europe at the beginning of 19 th century. The "Krakovo Gardens", in the city of Ljubljana in Slovenia, are one of the examples of the integration of cultivation farms in the cities. Krakovo gardens are constituted by an allotment system, and are situated in the urban area. These gardens were playing an important role as a source of fresh vegetable for the local people (naturalhomes.org, 2014). Shopping in Ljubljana's green market on Saturday mornings 


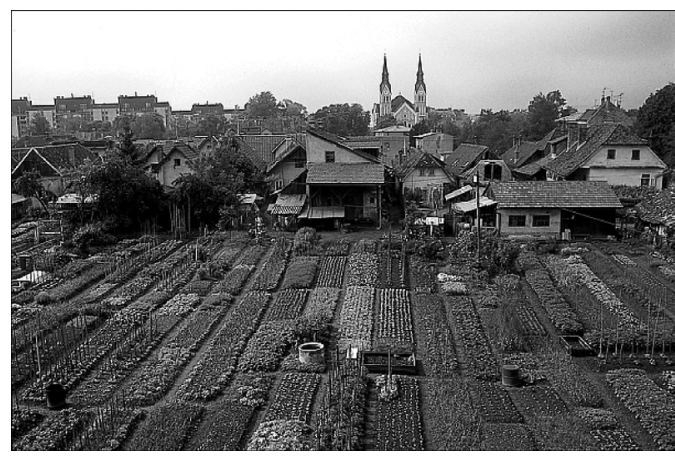

Figure 1. The "Krakovo Gardens" in Ljubljana, Slovenia (naturalhomes.org).

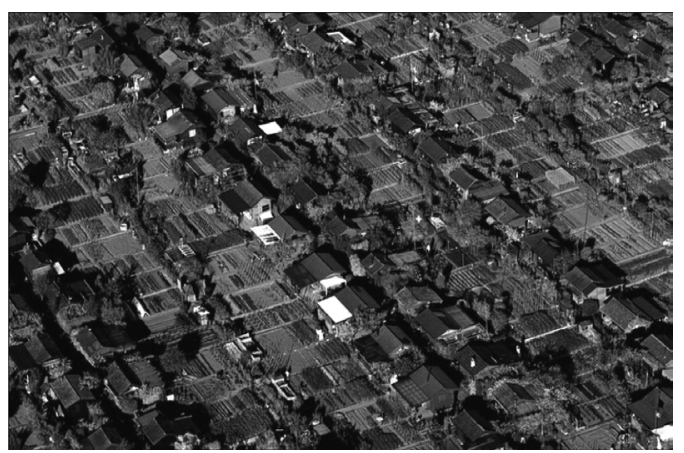

Figure 2. The "Schrebergarten" in Geneva, Switzerland (naturalhomes.org).

was a community experience where people bought fresh organic food grown within a stone's throw of where they lived (Fig. 1).

Another European example of peri-urban agriculture is "Schrebergarten" (Schreber gardens) in Geneva, Switzerland. The founder of these types of gardens was a 19th century German physician called Moritz Schreber. The idea of organised allotment gardening reached a first peak after 1864, when Schreber started the 'Schreber Movement' in Leipzig where areas within the city were made available for children to play in a healthy environment in harmony with nature. Later on these areas included gardens for children, but soon adults began to cultivate them (Fig. 2). This kind of gardening later became popular in other European countries such as Austria and Switzerland (naturalhomes.org, 2014).

Increasing climate change effects on urban areas bring out much vulnerability related to the natural hazards such as droughts and floods, which have direct negative consequences on rural agriculture like lack of food. Peri-urban agriculture represents an efficient strategy for emergency food supply and the mitigation of food insecurity. In addition to these advantages, it offers opportunities of employments in farming sector to the households of per-urban area. As a socio-cultural issue peri-urban agriculture supports the development of local products so eventually contributes to the local economies and encourages the transmission of collective food production culture to the future generations.

\section{CASE STUDY: ECOCITY IN CENAIA, PISA}

Since 2008, the Faculty of Architecture of Florence University has released the Laboratory AMA (Architecture, Materials, Environment) that aims for the realization of architectural projects that has as its fundamental principle the interpretation of the contemporary architectural languages by the use of earth and other materials with low environmental impact. The activities of laboratory AMA develop both theoretical and practical levels through workshops in situ to facilitate a better understanding of the local culture, traditional construction systems while providing interaction with its communities.

In 2013, one of the design themes of Laboratory AMA has been "Ecocity project in Cenaia" in the province of Pisa, Italy. This theme was proposed by a local group of citizens named "Ecocity" who aimed to develop a project to be included in the existing village of Cenaia financed by private funds and capable to reorganize the urban structure and give a new identity to the settlement. The goal of the Ecocity was to realize an experimental cohousing and cofarming village by creating a group of residential units with the spaces for productive, cultural and touristic facilities related with lands for farming activities and using local materials particularly rammed earth.

\subsection{Urban development proposal: Co-housing -and Co-farming in Cenaia}

The verification tool of the project was the planning of new built-in functions as a part of city that can act as a paradigm of strategic rethinking the future development of the entire Cenaia, including redevelopment of the formless forbearing fraying building and visionary projection in a broader unified design. The solutions in common is the placement in the agricultural belt near the village (Fig. 3 and 4), and the use of the technology of rammed earth, chosen as the construction practice of surprising and radical modernity in terms of sustainability and also culture. This is a type of technology that, thanks to the articulated functional mixture of Ecocity, also demonstrates an effective compositional and expressive ductility facing needs, feelings and language of a full contemporary architecture. 


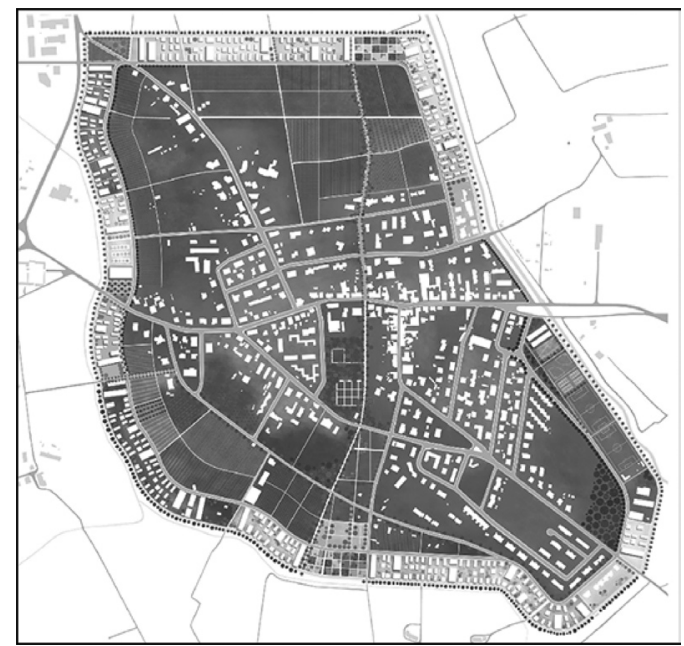

Figure 3. The town of Cenaia and the new limits: the Ecocity Project (G. Aguti, G. Boscherini).

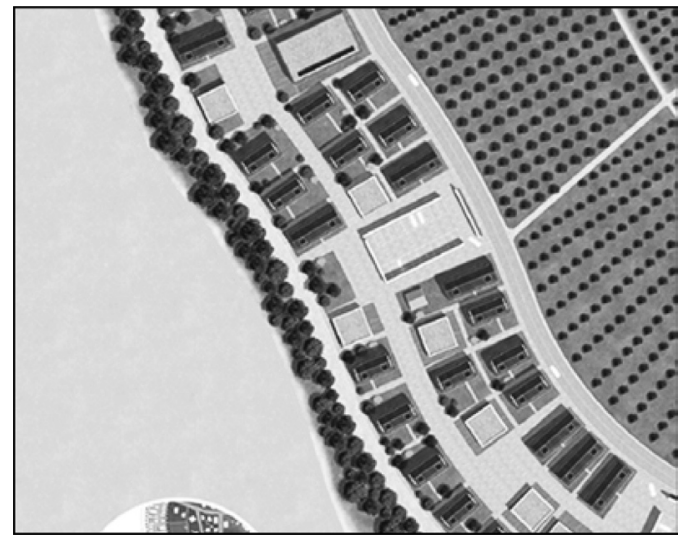

Figure 4. The Ecoctiy project: Masterplan (G. Aguti, G. Boscherini).

\subsection{Design concept I: New modes of living urban fringe}

In the hypothesis of Ecocity the act of living, teaching, recreational, cultural and social activities are intertwined especially with unusual forms of work and production in the agricultural sector, which identify in urban perimeter the most appropriate site to organize forms of relationships and innovative settlement. In this area of linkages as changing characteristics between town and country, the integration of the activities of life becomes the key to a social community (Fig. 5). The development of new ways for the type-morphologies of the buildings, their necessity and spatial solutions, size and scale of the space and individual, social and public, (both productive and receptive-commercial) connotes the research of evolutionary standards based on hybridization-fusion of nature and earthen architecture.

\subsection{Design concept II: Peri urban agricultural landscape as a new limit for the urban footprint}

After receiving the request of projecting Ecocity in the peri-urban area of Cenaia, the solution developed by a thesis has interpreted it as a trigger and plug of some kind of new urban walling. Inspired by the idea of returning to manage and compose the city limits to obtain a categorical clarity between internal and external in terms of stopping the construction colonization of the countryside and landscape, the conceptual appeal to the ancient city walls assume a destiny of the peri-urban fringe as a urban facade, subject to re-design in the sense of ability to complete form. And just the hybrid nature of the urban fringe inside the walls of the past, that blended organically residences, convents, barracks and factories with large areas of cultivated zones, both private and for collective security, supports the hypothesis of a contemporary high-mix functionalism.

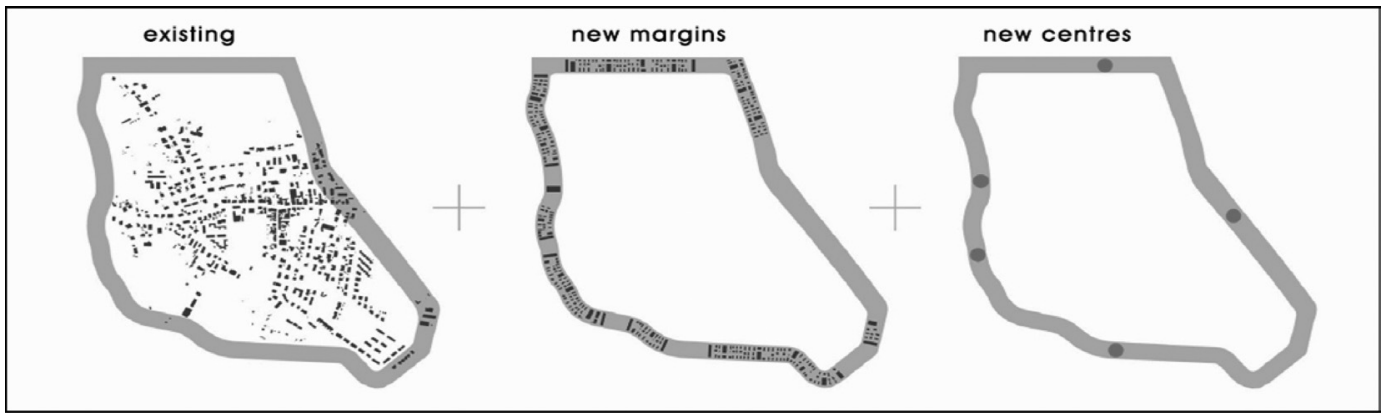

Figure 5. The Ecoctiy project: Existing and new building system (G. Aguti, G. Boscherini). 


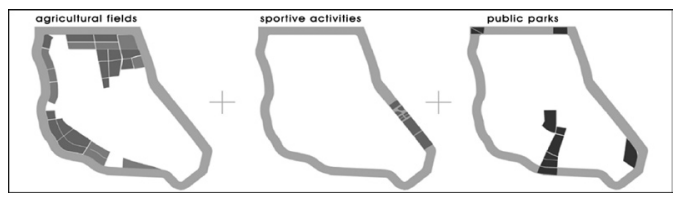

Figure 6. The Ecoctiy project: Green areas system (G. Aguti, G. Boscherini).

Similarly, the new walls along the edge of Cenaia suburban take on the role of containment building expansion according to the project-controlled characters of an urban and architectural system unitary limit, a clear separation between internal and external sides of the urban areas. As a kind of hoops of consolidation and adjustment of feathering buildings, the new walls are proposed as a form of precise identification of agglomeration in the landscape, through specific geometries from the schedule of natural elements, sediments, signs and intentions. This become a strong urban structure, that in this condition the urban fringe identifies the contamination of the state, in which the opposition between rural and urban condition can reinvent the third phenomenology of a "urb-agricolo" landscape defined and envisioned as a band of intervening permeable, functionally and socially highly specialized, the territory of reconciliation and restoration of the form and content between city and pure countryside (Fig. 6).

In foreshadowing of the thesis, the present perimeter system of the city is finally re-founded and got a future image as a unit consisting of a sequence of joints, in a sort of game of dominoes whose pieces combine to give the general identity as a whole and consonance, from which to start towards the city center with detailed practical remedial for rehabilitation, integration and densification. Among them, new and specific modes of the role of shared agricultural cultivation addresses to the reconstitution of a plausible progressive and ecological collective life.

\section{CONCLUSIONS}

Urban fringe manifests coexistence and mixed characteristics of urban and rural. This complexity requires multidimensional approaches and better understandings for the linkage development strategies. In this case peri-urban agriculture appears as the most appropriate solution as it responds to the needs of urban systems through elaborating the potentials that rural areas offer.

Peri-urban agriculture contributes to revitalize the urban fringe and makes it a more frequented place by its inhabitants so it saves urban fringe from being like a desert. It helps increasing the collectiveness and also self-sustainability. As seen in the case study of Cenaia, the functionality of peri-urban agriculture has a great capacity of compatibility with recreational and touristic activities. Therefore, the integration of urban-characterised functions, such as tourism and commercial, with the rural-characterised functions like agriculture may create a good strategic solution that both urban and rural can benefit. Another factor that encourages peri-urban agriculture is its relevant role in food insecurity of the cities. As men-tioned before, with the "proximity" to the city and "lowdependence" on the transportation, peri-urban agriculture becomes a favourable strategy for the development of the cities, above all Pisa.

This paper aims to emphasize the substantial role of peri-urban agriculture in creating strong and compatible linkage strategies between cities and rural areas. One of the purposes of this research is to identify the characteristics of urban fringe and how peri-urban agriculture meets the needs of this given zone. This paper also points out the adaptability of integration of peri-urban agriculture with different attractive urban functions to revitalize the urban fringe through a case study from Cenaia, Pisa. Considering its social, socioeconomic and environmental benefits, peri-urban agriculture should be recognised and integrated by urban planners to the development of contemporary urbanism.

\section{REFERENCES}

Caruso, G. 2001. Peri-urbanisation: The situation in Europe: A bibliographical note and survey of studies in the Netherlands, Belgium, Great Britain, Germany, Italy and the Nordic countries. Report prepared for DATAR, France.

Errington, A. 1994. The peri-urban fringe: Europe's forgotten rural areas. Journal of Rural Studies 10(4):367-375.

FAQ. 1999. Urban and Peri-urban agriculture. (Online) Available:http://www.fao.org/unfao/bodies/coag/ coag15/x0076e.htm\#P26_252. Accessed: March 2014.

Griffiths, M.B. 2010. Lamb Buddha's Migrant Worksers: Self assertion on China's urban fringe. Journal of Current Chinese Affairs (China Aktuell) 39(2): 3-37.

Mitchell, C.J.A. 2004. Making sense of counter-urbanisation. Journal of Rural Studies 20(1): 15-34.

Opkala, D.C.L. 2003. Promoting the positive rural-urban linkages approach to sustainable development and employment creation: The role of UN-HABITAT, FIG Regional Conference 2.

Theobald, P. 1988. Districts on the edge: The impact of urban sprawl on a rural community. Research in Rural Education 5(2).

Zasada, I., Fertner, C., Piorr, A., Nielsen, T.S. 2011. Peri urbanisation and multi-functional adaptation of agriculture around Copenhagen. Danish Journal of Geography $111(1): 59-72$. 